\title{
THEORY OF MIMO BIORTHOGONAL PARTNERS AND THEIR APPLICATION IN CHANNEL EQUALIZATION
}

\author{
Bojan Vrcelj and P. P. Vaidyanathan \\ Dept. of Electrical Engr. 136-93, Caltech, Pasadena, CA 91125, USA \\ E-mail: bojan@systems.caltech.edu,ppvnath@sys.caltech.edu
}

\begin{abstract}
Channel equalization is an important step in most applications of digital communications. In this paper we consider the equalization of Multiple Input Multiple Output (MIMO) channels. To that end we derive the theory of MIMO biorthogonal partners, a concept that has already been introduced (only in the scalar case) by the authors. We develop conditions for the existence of an FIR MIMO biorthogonal partners and describe their application in the MIMO channel equalization. We also show that it is possible to exploit the non-uniqueness of FIR MIMO biorthogonal partners in order to design flexible fractionally spaced MIMO equalizers that will be more robust to the channel noise. ${ }^{1}$
\end{abstract}

\section{INTRODUCTION}

Digital filters $H(z)$ and $F(z)$ are called biorthogonal partners of each other with respect to an integer $M$ if their cascade $H(z) F(z)$ obeys the Nyquist $(M)$ property [1]. The theory of biorthogonal partners was developed recently in [1] for the simple, single input single output (SISO) case. Multiple input multiple output (MIMO) biorthogonal partners are defined using a similar approach. One major difference is readily observed: because of the properties of matrix multiplication, the "biorthogonal partner" relation is not symmetric in the MIMO case. In particular this implies that in the MIMO case we have to distinguish between a left biorthogonal partner (LBP) and a right biorthogonal partner (RBP).

Vector signals naturally occur in several applications such as antenna arrays in mobile communications. We will consider the FIR MIMO biorthogonal partners in greater detail. In this paper we will extend some of the most important results on biorthogonal partners, derived in [1] for the scalar case, to the case of vector signals. A set of necessary and sufficient conditions on a transfer matrix $\mathbf{F}(z)$ such that there exists an FIR MIMO biorthogonal partner $\mathbf{H}(z)$ will be derived. We will also concentrate on the fact that the FIR MIMO biorthogonal partner (if it exists) is not unique. We will exploit this non-uniqueness in order to reduce the noise power at the output of the equalizer. Finally, we will address the performance of our algorithms through a simple example of a fractionally spaced equalizer (FSE) for a MIMO channel. The example will demonstrate how it is possible to equalize an ill-conditioned

\footnotetext{
${ }^{1}$ Work supported in part by National Science Foundation under Grant MIP 0703755
}

MIMO channel using the FSE and how its performance can be improved by exploiting the non-uniqueness of that solution.

\section{I-A Notations}

If not stated otherwise, all notations are as in [2]. We use the notation $[x(n)]_{\downarrow M}$ and $[X(z)]_{\downarrow M}$ to denote the decimated version $x(M n)$ and its $z$-transform. The expanded version

$$
\begin{cases}x(n / M) & \text { for } n=\operatorname{mul} \text { of } M \\ 0 & \text { otherwise }\end{cases}
$$

is similarly denoted by $[x(n)]_{\uparrow M}$, and its $z$-transform $X\left(z^{M}\right)$ denoted by $[X(z)]_{\uparrow M}$. In a block diagram, the scalar decimation and expansion operations will be marked by encircled symbols $\downarrow M$ and $\uparrow M$ respectively. In the case of vectors and matrices, the decimation and expansion are performed on each element separately. The corresponding symbols in this case are placed in square boxes. The polyphase decomposition [2] is also valid in the matrix case. Thus for example if $\mathbf{F}(z)$ is a matrix transfer function, then it can be written in the Type- 2 polyphase form as

$$
\mathbf{F}(z)=\sum_{k=0}^{M-1} z^{k} \mathbf{F}_{k}\left(z^{M}\right) .
$$

\section{MIMO BIORThOgONAL PARTNERS: DEFINITION AND PROPERTIES}

We start the discussion in this section by formally defining the notion of a MIMO biorthogonal partner.

Definition 1. MIMO Biorthogonal partners. A MIMO transfer function $\mathbf{H}(z)$ is said to be a left biorthogonal partner (LBP) of $\mathbf{F}(z)$ with respect to an integer $M$ if

$$
[\mathbf{H}(z) \mathbf{F}(z)]_{\downarrow M}=\mathbf{I} .
$$

Similarly, a MIMO transfer function $\mathbf{H}(z)$ is said to be a right biorthogonal partner (RBP) of $\mathbf{F}(z)$ with respect to an integer $M$ if $[\mathbf{F}(z) \mathbf{H}(z)]_{\downarrow M}=\mathbf{I}$.

The interpretation of the first part of the above definition is shown in Fig. 1. It can be seen that if $\mathbf{H}(z)$ is a LBP of $\mathbf{F}(z)$ that implies that $\mathbf{F}(z)$ is a RBP of $\mathbf{H}(z)$, but it does not imply that $\mathbf{H}(z)$ is also a RBP of $\mathbf{F}(z)$. The latter would happen if, for example, the two matrices commuted. The other important point to make here is that if $M$ is changed, the two filters might not remain partners. However, we will often omit the term "with respect to $M$ ", since it will usually be understood from the context. 


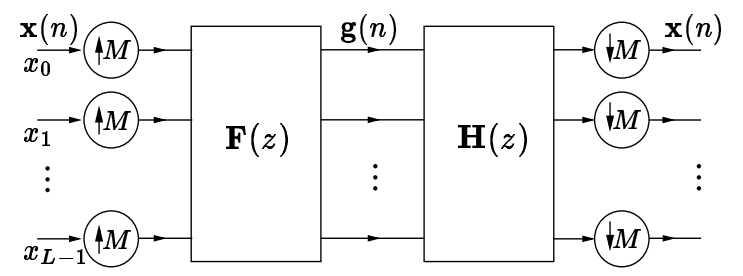

Figure 1: Block diagram interpretation of a left biorthogonal partner.

In the following we present two important theorems. The first one gives the most general form of biorthogonal partners. The second states the condition on a transfer matrix $\mathbf{F}(z)$ and the integer $M$ such that there exists an FIR biorthogonal partner. A way of constructing that biorthogonal partner will also be described.

Theorem 1. General form of biorthogonal partner.

1. A MIMO transfer function $\mathbf{H}(z)$ is a LBP of $\mathbf{F}(z)$ if and only if it can be expressed in the form

$$
\mathbf{H}(z)=\left([\mathbf{G}(z) \mathbf{F}(z)]_{\downarrow M \uparrow M}\right)^{-1} \mathbf{G}(z)
$$

for some MIMO transfer function $\mathbf{G}(z)$.

2. A MIMO transfer function $\mathbf{H}(z)$ is a $\operatorname{RBP}$ of $\mathbf{F}(z)$ if and only if it can be expressed in the form

$$
\mathbf{H}(z)=\mathbf{G}(z)\left([\mathbf{F}(z) \mathbf{G}(z)]_{\downarrow M \uparrow M}\right)^{-1}
$$

for some MIMO transfer function $\mathbf{G}(z)$.

Example 1. If $\left|\operatorname{det}\left[\mathbf{F}\left(e^{j \omega}\right)\right]\right|>0$ for all $\omega$ then $\mathbf{H}(z)=$ $\mathbf{F}^{-1}(z)$ is a theoretically stable biorthogonal partner (both LBP and RBP) of $\mathbf{F}(z)$. It can be obtained from (3) or (4) with the choice $\mathbf{G}(z)=\mathbf{F}^{-1}(z)$. This is conceptually the simplest biorthogonal partner.

Proof of Theorem 1. First we will prove the "if part" of the statement one. Given $\mathbf{H}(z)$ of the above form (3), we have

$$
\begin{aligned}
{[\mathbf{H}(z) \mathbf{F}(z)]_{\downarrow M} } & =\left[\left([\mathbf{G}(z) \mathbf{F}(z)]_{\downarrow M \uparrow M}\right)^{-1} \mathbf{G}(z) \mathbf{F}(z)\right]_{\downarrow M} \\
& =\left([\mathbf{G}(z) \mathbf{F}(z)]_{\downarrow M}\right)^{-1}[\mathbf{G}(z) \mathbf{F}(z)]_{\downarrow M}=\mathbf{I} .
\end{aligned}
$$

The "if part" of the second statement follows in the same manner. Now we will prove the "only if part" of the second statement. For this, first consider Fig. 2(a). Here $\mathbf{x}_{i}(n)$ is an arbitrary vector sequence and $\mathbf{g}_{i}(n)$ is the corresponding output of $\mathbf{H}(z)$. By assumption $\mathbf{H}(z)$ is a RBP of $\mathbf{F}(z)$ and from the definition we have that the output of the system has to be $\mathbf{x}_{i}(n)$ again. However, this also means that the signal $\mathbf{g}_{i}(n)$, when input to the system of Fig. 2(b), comes out as $\mathbf{g}_{i}(n)$. Thus we have

$$
\mathbf{H}(z)\left[\mathbf{F}(z) \mathbf{G}_{i}(z)\right]_{\downarrow M \uparrow M}=\mathbf{G}_{i}(z) .
$$

This equality holds for any $\mathbf{G}_{i}(z)$ obtained as in Fig. 2(a). We repeat the procedure sufficient number of times, each time taking $\mathbf{X}_{n}(z)$ to be linearly independent from the previous vectors $\mathbf{X}_{1}(z), \mathbf{X}_{2}(z), \ldots \mathbf{X}_{n-1}(z)$. Collecting those vectors as columns in a matrix $\mathbf{X}(z)$, and the corresponding vectors $\mathbf{G}_{i}(z)$ in a matrix $\mathbf{G}(z)$, we have the following

$$
\mathbf{H}(z)[\mathbf{F}(z) \mathbf{G}(z)]_{\downarrow M \uparrow M}=\mathbf{G}(z),
$$

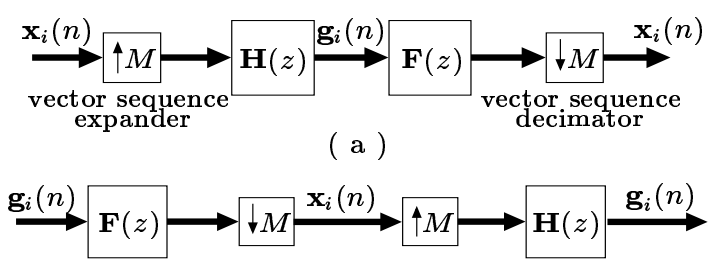

( b )

Figure 2: Pertaining to the proof of Theorem 1.

which after solving for $\mathbf{H}(z)$ gives

$$
\mathbf{H}(z)=\mathbf{G}(z)\left([\mathbf{F}(z) \mathbf{G}(z)]_{\downarrow M \uparrow M}\right)^{-1}
$$

and this concludes the proof of (4). Notice that

$$
[\mathbf{F}(z) \mathbf{G}(z)]_{\downarrow M \uparrow M}=[\mathbf{X}(z)]_{\uparrow M},
$$

so that by choosing the sequences $\mathbf{x}_{i}(n)$ carefully we can ensure that the matrix inversion in (7) is valid. Now we move on to prove the "only if part" of the first statement. For this we notice that if $\mathbf{H}(z)$ is a LBP of $\mathbf{F}(z)$, then $\mathbf{H}^{T}(z)$ is a $\operatorname{RBP}$ of $\mathbf{F}^{T}(z)$, with the superscript $T$ denoting the transpose of a matrix. Thus from (4) we have

$$
\mathbf{H}^{T}(z)=\mathbf{G}^{T}(z)\left(\left[\mathbf{F}^{T}(z) \mathbf{G}^{T}(z)\right]_{\downarrow M \uparrow M}\right)^{-1}
$$

for some matrix $\mathbf{G}^{T}(z)$. Finally, taking the transpose of both sides we arrive at (3) and this concludes the proof. $\quad \nabla \nabla \nabla$

In the proof of Theorem 1 we used the idea of "transposing the result" for RBP in order to prove a similar result for LBP. The same trick could also be used for the remaining results in the paper. That is why we will consider only left biorthogonal partners in the following; very similar results hold for right biorthogonal partners. Theorem 1 gives the most general form of a biorthogonal partner. Since that form includes matrix inversion, the resulting MIMO filter will often be IIR or even unstable depending on the matrix $\mathbf{G}(z)$. One important issue therefore is how to find an FIR biorthogonal partner and under what conditions it is possible to find one. In the next theorem we address this issue. In the following, the symbol $\operatorname{grcd}[\cdot]$ represents the greatest right common divisor [3],[2].

Theorem 2. Existence of FIR LBP. Suppose F $(z)$ is causal and FIR, given by the Type-2 polyphase form as in (1). Then there exists a causal FIR matrix $\mathbf{H}(z)$ such that

$$
[\mathbf{H}(z) \mathbf{F}(z)]_{\downarrow M}=\mathbf{I}
$$

if and only if $\operatorname{grcd}\left[\mathbf{F}_{0}(z), \mathbf{F}_{1}(z), \ldots \mathbf{F}_{M-1}(z)\right]$ is a unimodular ${ }^{2}$ matrix $\mathbf{R}(z)$.

Example 2. Given an arbitrary MIMO transfer function, the grcd-condition is almost always satisfied. For example let

$$
\mathbf{F}(z)=\left[\begin{array}{cc}
3+2 z^{-1}+z^{-2} & 2+3 z^{-1}+z^{-2} \\
1+3 z^{-2} & 2+z^{-1}+3 z^{-2}
\end{array}\right]
$$

\footnotetext{
${ }^{2} \mathrm{~A}$ polynomial matrix is said to be unimodular if its determinant is a nonzero constant.
} 
The trivial biorthogonal partner (as in Example 1) is IIR in this case, since $\operatorname{det}[\mathbf{F}(z)]=4+4 z^{-1}+6 z^{-2}-2 z^{-3}$. However, it can be verified that the grcd of the polyphase components (of order 2) of $\mathbf{F}(z)$ is unimodular, with one solution being

$$
\mathbf{R}(z)=-2\left[\begin{array}{ll}
4 & 2 \\
0 & 1
\end{array}\right] .
$$

Therefore, an FIR LBP indeed exists and one possibility is

$$
\mathbf{H}(z)=\frac{1}{16}\left[\begin{array}{cc}
8+4 z^{-1}+3 z^{-2} & -8-z^{-2} \\
-4-8 z^{-1}-6 z^{-2} & 12+2 z^{-2}
\end{array}\right] .
$$

Before proceeding to the proof of Theorem 2, several comments are due. Here we consider the case where $\mathbf{F}(z)$ is a square matrix, $L \times L$ say. This is done mostly for simplicity and it can be shown that a more general, rectangular version of the theorem also holds (with some restrictions). The constraint on $\mathbf{F}(z)$ and its LBP to be causal is also unnecessary; it can be avoided if we allow the determinant of $\mathbf{R}(z)$ to be a delay rather than a constant.

Proof of Theorem 2. First we consider the case $M=2$. If $\mathbf{F}_{0}(z)$ and $\mathbf{F}_{1}(z)$ are right coprime (which is equivalent to saying that $\mathbf{R}(z)$, i.e. the $\operatorname{grcd}\left[\mathbf{F}_{0}(z), \mathbf{F}_{1}(z)\right]$ is unimodular) then there exist polynomial matrices $\mathbf{H}_{0}(z)$ and $\mathbf{H}_{1}(z)$ such that

$$
\mathbf{H}_{0}(z) \mathbf{F}_{0}(z)+\mathbf{H}_{1}(z) \mathbf{F}_{1}(z)=\mathbf{I} .
$$

This follows from the simple Bezout identity [3]. In fact, from the construction for a grcd (also in [3]) it follows that there exists a unimodular matrix $\mathbf{U}(z)$ such that

$$
\underbrace{\left[\begin{array}{ll}
\mathbf{U}_{11}(z) & \mathbf{U}_{12}(z) \\
\mathbf{U}_{21}(z) & \mathbf{U}_{22}(z)
\end{array}\right]}_{\mathbf{U}(z)}\left[\begin{array}{l}
\mathbf{F}_{0}(z) \\
\mathbf{F}_{1}(z)
\end{array}\right]=\left[\begin{array}{c}
\mathbf{R}(z) \\
\mathbf{0}
\end{array}\right],
$$

where all the building blocks above are $L \times L$ matrices and $\mathbf{U}(z)$ is a $2 L \times 2 L$ matrix. Now we see that in this case we can choose

$$
\mathbf{H}_{0}(z)=\mathbf{R}^{-1}(z) \mathbf{U}_{11}(z), \mathbf{H}_{1}(z)=\mathbf{R}^{-1}(z) \mathbf{U}_{12}(z)
$$

and that those are indeed polynomial (actually causal FIR) matrices since $\mathbf{R}(z)$ is unimodular. Extension to arbitrary $M$ follows readily by applying the rule

$$
\begin{aligned}
& \operatorname{grcd}_{0 \leq k \leq M-1}\left[\mathbf{F}_{k}(z)\right]= \\
& \quad \operatorname{grcd}\left[\mathbf{F}_{M-1}(z), \operatorname{grcd}_{0 \leq k \leq M-2}\left[\mathbf{F}_{k}(z)\right]\right] .
\end{aligned}
$$

Now, suppose by contradiction that $\mathbf{F}(z)$ has a causal FIR LBP $\mathbf{H}(z)$, but that $\operatorname{grcd}\left[\mathbf{F}_{0}(z), \mathbf{F}_{1}(z), \ldots \mathbf{F}_{M-1}(z)\right]=\mathbf{C}(z)$ is not unimodular. Writing $\mathbf{H}(z)$ in the Type-1 polyphase form we have

$$
\begin{aligned}
\mathbf{I} & =[\mathbf{H}(z) \mathbf{F}(z)]_{\downarrow M}=\sum_{k=0}^{M-1} \mathbf{H}_{k}(z) \mathbf{F}_{k}(z) \\
& =\left(\sum_{k=0}^{M-1} \mathbf{H}_{k}(z) \hat{\mathbf{F}}_{k}(z)\right) \mathbf{C}(z)
\end{aligned}
$$

and it follows that

$$
\sum_{k=0}^{M-1} \mathbf{H}_{k}(z) \hat{\mathbf{F}}_{k}(z)=\mathbf{C}^{-1}(z) .
$$

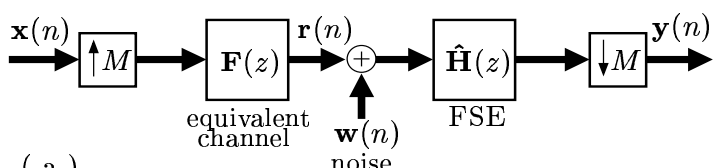

( a )

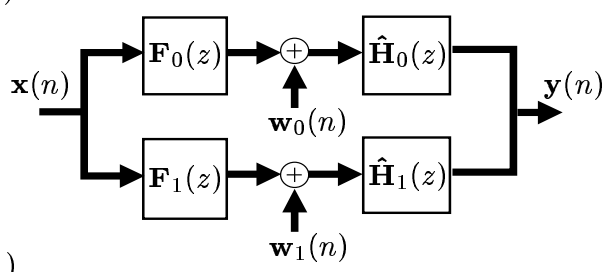

( b )

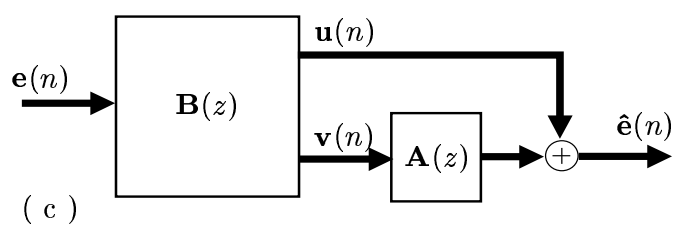

Figure 3: Block diagram interpretation of the construction of FSE. (a) Discrete-time equivalent communication channel with FSE, (b) equivalent of (a) obtained using noble identities, and (c) equivalent model for noise.

The left hand side of the above equation is a causal FIR matrix (since all $\mathbf{H}_{k}(z)$ and $\hat{\mathbf{F}}_{k}(z)$ are causal FIR), but the right hand side is not. This contradiction concludes the proof. $\quad \nabla \nabla \nabla$

It is important to notice here that, if it exists, FIR LBP is not unique. There are two reasons for this. Firstly, grcd of two matrices is unique only up to a premultiplication by a unimodular matrix. Secondly, there are many pairs of matrices $(\mathbf{U}(z), \mathbf{R}(z))$ that satisfy (8) and each of them provides a valid solution. In the next section we will consider the case where an FIR LBP is used as a MIMO channel equalizer. We will show that this flexibility in the choice of $\mathbf{H}(z)$ can be exploited in order to reduce the undesirable amplification of the noise at the receiver.

\section{OPTIMIZING LBP FOR CHANNEL EQUALIZATION}

Consider the discrete-time equivalent of a MIMO digital communication channel with a fractionally spaced equalizer (FSE) [4],[5] shown in Fig. 3(a). For simplicity we will assume that the equivalent channel $\mathbf{F}(z)$ is a square $(L \times L)$ matrix and that the oversampling ratio $M$ is equal to 2 . In this case the channel from Fig. 3(a) can be redrawn as in Fig. 3(b). Here $\mathbf{w}_{0}(n)$ and $\mathbf{w}_{1}(n)$ are the corresponding polyphase components of the noise vector sequence $\mathbf{w}(n)$ from Fig. 3(a) and $\hat{\mathbf{H}}_{0}(z)$ and $\hat{\mathbf{H}}_{1}(z)$ are the polyphase components of a FSE. Recall that if the conditions of Theorem 2 are satisfied, than $\mathbf{H}_{0}(z)$ and $\mathbf{H}_{1}(z)$ as in (9) lead to one possible solution for $\hat{\mathbf{H}}(z)$. However, from (8) we see that another class of solutions is given by

$$
\begin{aligned}
\hat{\mathbf{H}}_{0}(z) & =\mathbf{H}_{0}(z)+\mathbf{A}(z) \mathbf{U}_{21}(z) \\
\hat{\mathbf{H}}_{1}(z) & =\mathbf{H}_{1}(z)+\mathbf{A}(z) \mathbf{U}_{22}(z)
\end{aligned}
$$


for an arbitrary $L \times L$ matrix $\mathbf{A}(z)$. Our goal here is to design $\mathbf{A}(z)$ such that the noise component of $\mathbf{y}(n)$ is minimized. For that purpose, we consider the noise model shown in Fig. 3(b). Let us define the following

$$
\mathbf{e}(n) \triangleq\left[\begin{array}{c}
\mathbf{w}_{0}(n) \\
\mathbf{w}_{1}(n)
\end{array}\right], \mathbf{B}(z) \triangleq\left[\begin{array}{cc}
\mathbf{H}_{0}(z) & \mathbf{H}_{1}(z) \\
\mathbf{U}_{21}(z) & \mathbf{U}_{22}(z)
\end{array}\right] .
$$

Then the equivalent of the system from Fig. 3(b) is shown in Fig. 3(c). Therefore, our task now becomes that of finding the matrix $\mathbf{A}(z)=\sum_{i=0}^{N_{A}-1} \mathbf{A}_{i} z^{-i}$ such that the norm of

$$
\hat{\mathbf{e}}(n)=\mathbf{u}(n)+\sum_{i=0}^{N_{A}-1} \mathbf{A}_{\mathbf{i}} \mathbf{v}(n-i)
$$

is minimized. That turns out to be equivalent to the problem of finding the best linear estimator of order $N_{A}-1$ for the vector process $\mathbf{u}(n)$, given the observations $\mathbf{v}(n)$. The solution to this problem is well-known and is based on the orthogonality principle. Let us define the $L \times N_{A} L$ matrix $\mathcal{A}$ to be

$$
\mathcal{A} \triangleq\left[\begin{array}{lll}
\mathbf{A}_{0} & \mathbf{A}_{1} \ldots \mathbf{A}_{N_{A}-1}
\end{array}\right]
$$

and the $N_{A} L \times 1$ vector sequence $\mathcal{V}(n)$ to be

$$
\mathcal{V}(n) \triangleq\left[\mathbf{v}^{T}(n) \mathbf{v}^{T}(n-1) \ldots \mathbf{v}^{T}\left(n-N_{A}+1\right)\right]^{T} .
$$

Then by the orthogonality principle we have ${ }^{3}$

$$
E\left[\mathbf{u} \mathcal{V}^{\dagger}\right]+E\left[\mathcal{A} \mathcal{V} \mathcal{V}^{\dagger}\right]=\mathbf{0}
$$

so that the solution for the optimum $\mathcal{A}$ becomes

$$
\mathcal{A}=-E\left[\mathbf{u} \mathcal{V}^{\dagger}\right] \mathbf{R}_{\mathcal{V} \mathcal{V}}{ }^{-1},
$$

with $\mathbf{R}_{\mathcal{V} \mathcal{V}}$ denoting the autocorrelation of $\mathcal{V}$. This is nothing but the standard Wiener filter solution [6].

Next, we need to express the solution (13) in terms of the statistics of the input noise $\mathbf{e}(n)$. Define $\overline{\mathbf{H}}(z)(L \times 2 L$ matrix $)$ to consist of the first $L$ rows of the $2 L \times 2 L$ matrix $\mathbf{B}(z)$ defined in (11) and similarly $\overline{\mathbf{U}}(z)(L \times 2 L$ matrix $)$ to consist of the last $L$ rows of $\mathbf{B}(z)$. If $N_{B}-1$ is the order of $\mathbf{B}(z)$, we define $\overline{\mathbf{H}}_{i}$ and $\overline{\mathbf{U}}_{i}$ by

$$
\overline{\mathbf{H}}(z)=\sum_{i=0}^{N_{B}-1} \overline{\mathbf{H}}_{i} z^{-i} \text { and } \overline{\mathbf{U}}(z)=\sum_{i=0}^{N_{B}-1} \overline{\mathbf{U}}_{i} z^{-i} .
$$

Now, the $2 L\left(N_{A}+N_{B}-1\right) \times 1$ vector sequence $\mathcal{E}(n)$, the $L \times$ $2 L\left(N_{A}+N_{B}-1\right)$ matrix $\mathcal{H}$ and the $N_{A} L \times 2 L\left(N_{A}+N_{B}-1\right)$ matrix $\mathcal{U}$ are defined as follows

$$
\begin{aligned}
& \mathcal{E}(n) \triangleq\left[\mathbf{e}^{T}(n) \mathbf{e}^{T}(n-1) \ldots \mathbf{e}^{T}\left(n-N_{A}-N_{B}+2\right)\right]^{T} \\
& \mathcal{H} \triangleq\left[\begin{array}{lll}
\overline{\mathbf{H}}_{0} & \overline{\mathbf{H}}_{1} \ldots \overline{\mathbf{H}}_{N_{B}-1} & \mathbf{0} \ldots \mathbf{0}
\end{array}\right] \\
& \mathcal{U} \triangleq\left[\begin{array}{cccccc}
\overline{\mathbf{U}}_{0} & \ldots & \overline{\mathbf{U}}_{N_{B}-1} & \mathbf{0} & \ldots & \mathbf{0} \\
\mathbf{0} & \overline{\mathbf{U}}_{0} & \ldots & \overline{\mathbf{U}}_{N_{B}-1} & \ldots & \mathbf{0} \\
\vdots & & \ddots & & \ddots & \\
\mathbf{0} & \ldots & \mathbf{0} & \overline{\mathbf{U}}_{0} & \ldots & \overline{\mathbf{U}}_{N_{B}-1}
\end{array}\right]
\end{aligned}
$$

${ }^{3}$ Symbol $E[\cdot]$ denotes the expected value.
Then the following holds

$$
\mathbf{u}(n)=\mathcal{H} \mathcal{E}(n), \quad \mathcal{V}(n)=\mathcal{U} \mathcal{E}(n) .
$$

Finally, all we need to do is substitute (14) in (13) and arrive at the final solution for $\mathcal{A}$ :

$$
\mathcal{A}=-\mathcal{H} \mathbf{R}_{\mathcal{E} \mathcal{E}} \mathcal{U}^{\dagger}\left(\mathcal{U} \mathbf{R}_{\mathcal{E} \mathcal{E}} \mathcal{U}^{\dagger}\right)^{-1}
$$

Notice that the final solution (15) depends only on the statistics of the input noise $\left(\mathbf{R}_{\mathcal{E E}}\right)$ and elements of the previously determined matrix $\mathbf{B}(z)$. Also, notice that (15) provides us with constant matrices $\mathbf{A}_{i}$ (as in (12)) and the linear estimator $\mathbf{A}(z)$ is given by $\mathbf{A}(z)=\sum_{i=0}^{N_{A}-1} \mathbf{A}_{i} z^{-i}$. It should also be noted that the solution (10) is not the most general one, i.e. it is possible that there exists another FIR LBP $\mathbf{H}^{\prime}(z)$ which will outperform any $\hat{\mathbf{H}}(z)$ of the same order obtained via (10).

\section{EXPERIMENTAL RESULTS}

In this section we present the results of numerical simulations. Three different methods for the MIMO channel equalization are compared: symbol spaced equalization (SSE), FIR FSE as described in Sec. 2, and the generalized solution for FIR FSE as in (10) with $\mathbf{A}(z)$ chosen optimally for the given noise statistics. A block diagram of the digital communication system with the FSE was shown in Fig. 3(a). The equivalent block diagram for a system with the SSE is similar [4], except that there are no expanders and decimators. Also, in the SSE case the equivalent channel $\mathbf{F}_{2}(z)$ is obtained by decimating $\mathbf{F}(z)$ from Fig. 3(a):

$$
\mathbf{F}_{2}(z)=[\mathbf{F}(z)]_{\downarrow M} .
$$

Thus, the communication system with the SSE can be presented as a cascade of the equivalent channel $\mathbf{F}_{2}(z)$ and the equalizer. It follows that in order to equalize $\mathbf{F}_{2}(z)$ perfectly using (the zeroforcing) SSE, we need to invert it

$$
\mathbf{H}_{S S E}(z)=\mathbf{F}_{2}^{-1}(z) \text {. }
$$

Although in the matrix case it is still possible to end up with an FIR SSE, in general we have no control over the behavior of the inverse in (16), so it can be IIR or even unstable. In the following example we show how this problem can be treated using FSEs. Moreover, the nonuniqueness of the FSE based solution will be exploited in order to improve the receiver performance.

In our example we took $L=3$ and $M=2$. The MIMO channel $\mathbf{F}(z)$ was characterized by a $3 \times 3$ matrix polynomial of order 5 and the corresponding coefficients can be found at [7]. The constellation was chosen to be 16-QAM. The noise on the channel was taken to be white. The signal to noise ratio used in the experiments was obtained as (see Fig. 3(a))

$$
\mathrm{SNR}=20 \log _{10} \frac{\|\mathbf{r}\|_{2}}{\|\mathbf{w}\|_{2}} .
$$

The transfer function $\mathbf{F}_{2}(z)$ was chosen in such way that the inverse matrix $\mathbf{H}_{S S E}(z)$ is stable, but with two poles very close to the unit circle. In the absence of noise, SSE was performing as well as both the FSEs, i.e. all the symbols were received intact, but in the presence of noise the received symbols were almost un- 


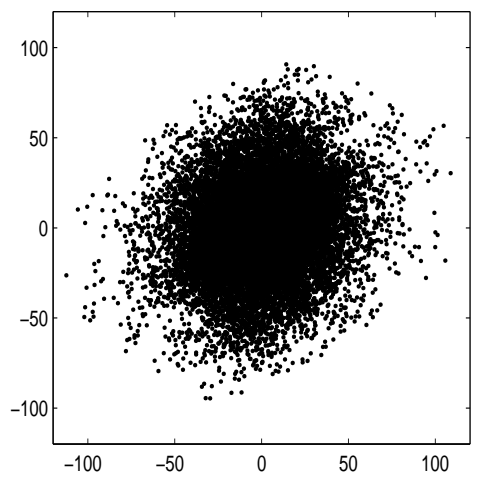

Figure 4: MIMO equalization: plain old SSE.

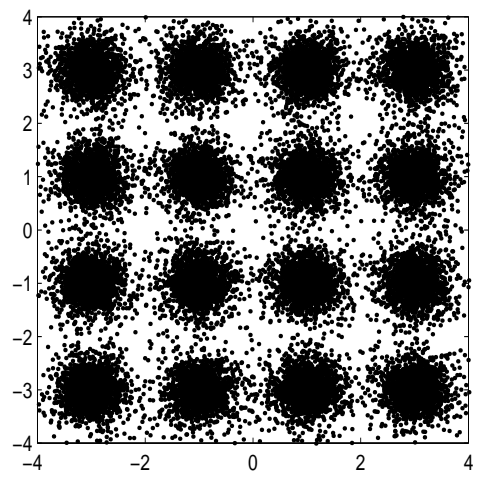

Figure 5: MIMO equalization: direct derivation of FSE.

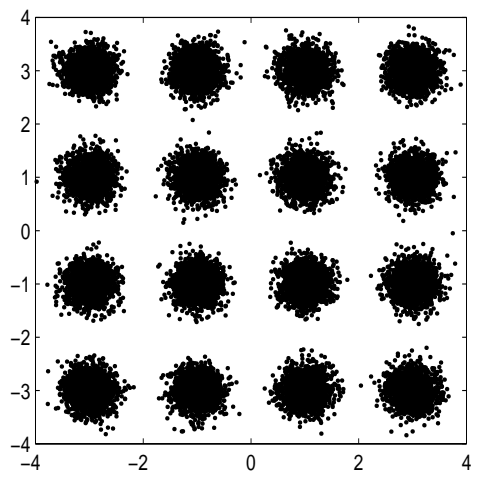

Figure 6: MIMO equalization: FSE optimized as in Sec. 3.

intelligible. To demonstrate this, in Fig. 4 we show the output of the SSE with SNR $=18 \mathrm{~dB}$. At the same noise level, a simple FIR FSE (Fig. 5) was already performing much better, with about eight out of $10^{3}$ symbols being misinterpreted. Finally, when we used the improved FIR FSE with only a third order estimator $\mathbf{A}(z)$ (the coefficients can also be found at [7]), the probability of error

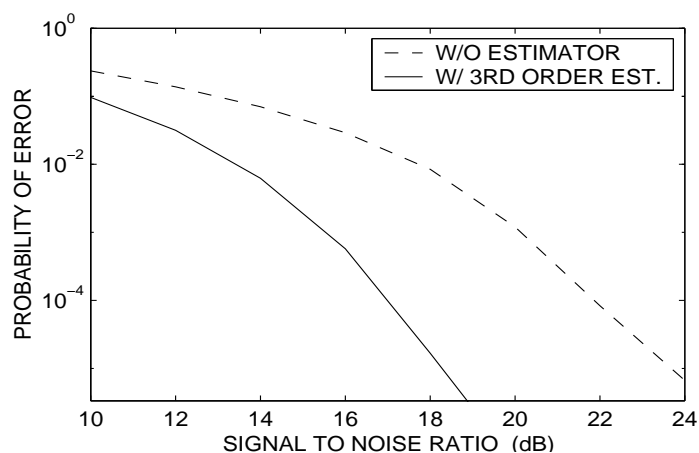

Figure 7: Probability of error vs. SNR: without estimator (dashed line) and with a third order estimator (solid line).

was reduced to $10^{-5}$ and the scatter plot in Fig. 6 shows clear separation.

In Fig. 7 we show the probability of error as a function of signal to noise ratio for the simple FIR FSE (i.e. with $\mathbf{A}(z)=\mathbf{0}$ ), and for the improved FIR FSE with the optimal third order estimator $\mathbf{A}(z)$. Those two cases correspond to Fig. 5 and Fig. 6, respectively. We can see that (in our example) at the error probability of $10^{-5}$, there is an improvement of approximately $5.5 \mathrm{~dB}$ in the equalizer performance, as the result of exploiting the redundancy in the design of the equalizer.

\section{CONCLUDING REMARKS}

The theory of MIMO biorthogonal partners is a natural extension of the SISO case considered in [1]. However, in the MIMO environment we are presented with additional degrees of freedom which may be useful in some applications. In this paper one such application was considered, namely the MIMO channel equalization. The possibilities for the improvement in the equalizer performance were demonstrated in the section with experimental results.

\section{REFERENCES}

[1] P. P. Vaidyanathan and B. Vrcelj, "Biorthogonal partners and applications," IEEE Trans. Signal Proc, in press.

[2] P. P. Vaidyanathan, Multirate Systems and Filter Banks, Prentice Hall, Inc., Englewood Cliffs, N.J.,1993.

[3] T. Kailath, Linear Systems, Prentice Hall, Inc., Englewood Cliffs, N.J., 1980.

[4] J. G. Proakis, Digital Communications, McGraw Hill, Inc., 1995.

[5] J. R. Treichler, I. Fijalkow and C. R. Johnson, Jr., "Fractionally spaced equalizers: how long should they really be?," IEEE Signal Processing Magazine, pp. 65-81, May 1996.

[6] S. Haykin, Adaptive Filter Theory. Prentice Hall, Inc., Upper Saddle River, N.J., 1996.

[7] http://www.systems.caltech.edu/dsp/students/bojan/papers/icc2001.html 